\title{
Supplementary Material for Spatial 3D Matérn priors for fast whole-brain fMRI analysis
}

\author{
Per Sidén, Finn Lindgren, David Bolin, Anders Eklund and Mattias Villani
}

\section{Derivation of the gradient and approximate Hessian}

For the optimization of the parameters $\boldsymbol{\theta}=\left\{\boldsymbol{\theta}_{s}, \boldsymbol{\lambda}, \mathbf{A}\right\}$, we need the gradient and approximate Hessian with respect to the different hyperparameters of the log marginal likelihood $\log p(\mathbf{y} \mid \boldsymbol{\theta})=\log p(\mathbf{y} \mid \boldsymbol{\beta}, \boldsymbol{\theta})+\log p(\boldsymbol{\beta} \mid \boldsymbol{\theta})-\log (\boldsymbol{\beta} \mid \mathbf{y}, \boldsymbol{\theta})$, which is constant with respect to $\boldsymbol{\beta}$. The gradient of the $\log$ posterior is then simply $\frac{\partial}{\partial \theta_{i}} \log p(\boldsymbol{\theta} \mid \mathbf{y})=\frac{\partial}{\partial \theta_{i}} \log p(\mathbf{y} \mid \boldsymbol{\theta})+$ $\frac{\partial}{\partial \theta_{i}} \log p(\boldsymbol{\theta})$, and similarly for the approximate Hessian. We will start this derivation by considering the $\log \operatorname{likelihood} \log p(\mathbf{y} \mid \boldsymbol{\beta}, \boldsymbol{\theta})$, then the conditional $\log$ posterior $\log (\boldsymbol{\beta} \mid \mathbf{y}, \boldsymbol{\theta})$, before producing the expressions for the log marginal likelihood gradient as well as the approximate log marginal likelihood Hessian. Finally, the corresponding posterior gradient and Hessian can be computed by adding the prior contributions derived in the last subsection.

To get a more robust optimization algorithm in practice, we use the reparameterizations $\tau_{0, k}=\log \left(\tau_{k}^{2}\right), \kappa_{0, k}=\log \left(\kappa_{k}^{2}\right)$ and $\lambda_{0, n}=\log \left(\lambda_{n}\right)$, so that the parameters are defined over the whole $\mathbb{R}$ and then perform the optimization over these new variables. The gradient for the new variables is easily obtained from the gradient for the old ones using the chain rule, for example $\frac{\partial \log p(\mathbf{y} \mid \boldsymbol{\theta})}{\partial \tau_{0, k}}=\frac{\partial \log p(\mathbf{y} \mid \boldsymbol{\theta})}{\partial \tau_{k}^{2}} \cdot \tau_{k}^{2}$. For $A_{p, n}$ we use the logit reparameterization $A_{0, p, n}=\log \left(\frac{1+A_{p, n}}{2}\right)-\log \left(\frac{1-A_{p, n}}{2}\right)$, guaranteeing $A_{p, n} \in(-1,1)$, which is the stability region for $\operatorname{AR}(1)$, and we have $\frac{\partial \log p(\mathbf{y} \mid \boldsymbol{\theta})}{\partial A_{0, p, n}}=\frac{\partial \log p(\mathbf{y} \mid \boldsymbol{\theta})}{\partial A_{p, n}} \cdot \frac{1-A_{p, n}^{2}}{2}$.

\subsection{Log likelihood}

As in Sidén et al. (2017, Appendix A), the log likelihood can be written as

$$
\log p(\mathbf{y} \mid \boldsymbol{\beta}, \boldsymbol{\theta})=\frac{T-P}{2} \sum_{n=1}^{N} \log \left(\lambda_{n}\right)-\frac{1}{2} \sum_{n=1}^{N} \lambda_{n} l_{n}\left(\mathbf{W}_{\cdot, n}\right)+\text { const },
$$

where

$$
l_{n}\left(\mathbf{W}_{\cdot, n}\right)=\mathbf{Y}_{\cdot, n}^{T} \mathbf{Y}_{\cdot, n}-2 \mathbf{Y}_{\cdot, n}^{T} \mathbf{X} \mathbf{W}_{\cdot, n}+\mathbf{W}_{\cdot, n}^{T} \mathbf{X}^{T} \mathbf{X} \mathbf{W}_{\cdot, n}
$$

in the case when the noise is independent over time and

$$
\begin{aligned}
l_{n}\left(\mathbf{W}_{\cdot, n}\right)= & \mathbf{Y}_{\cdot, n}^{T} \mathbf{Y}_{\cdot, n}-2 \mathbf{Y}_{\cdot, n}^{T} \mathbf{X} \mathbf{W}_{\cdot, n}+\mathbf{W}_{\cdot, n}^{T} \mathbf{X}^{T} \mathbf{X} \mathbf{W}_{\cdot, n}-2 \mathbf{Y}_{\cdot, n}^{T} \mathbf{d}_{n}^{T} \mathbf{A}_{\cdot, n}+\mathbf{A}_{\cdot, n}^{T} \mathbf{d}_{n} \mathbf{d}_{n}^{T} \mathbf{A}_{\cdot, n} \\
& +\mathbf{W}_{\cdot, n}^{T} \mathbf{B}_{n}^{T} \mathbf{A}_{\cdot, n}+\mathbf{A}_{\cdot, n}^{T} \mathbf{B}_{n} \mathbf{W}_{\cdot, n}-\mathbf{W}_{\cdot, n}^{T}\left(\mathbf{R} \mathbf{A}_{\cdot, n}+\left(\mathbf{R A} \mathbf{A}_{\cdot, n}\right)^{T}\right) \mathbf{W}_{\cdot, n} \\
& -\mathbf{A}_{\cdot, n}^{T}\left(\mathbf{D}_{n} \mathbf{W}_{\cdot, n}+\left(\mathbf{D}_{n} \mathbf{W}_{\cdot, n}\right)^{T}\right) \mathbf{A}_{\cdot, n}+\mathbf{W}_{\cdot, n}^{T}\left(\mathbf{A}_{\cdot, n}^{T} \mathbf{S} \mathbf{A}_{\cdot, n}\right) \mathbf{W}_{\cdot, n}
\end{aligned}
$$

(C) 0000 International Society for Bayesian Analysis

DOI: 0000 
when the noise follows an $\operatorname{AR}(P)$-process in each voxel with $\mathrm{AR}$-parameters $\mathbf{A}_{\cdot, n}$. We follow the notation in Sidén et al. (2017), except here $\boldsymbol{\beta}=\operatorname{vec}\left(\mathbf{W}^{T}\right)$. For convenience we list the different matrices and tensors and their sizes also here:

$$
\begin{aligned}
& \underset{P \times(T-P)}{\mathbf{d}_{n}} \text { contains lagged values of } \mathbf{Y}_{\cdot, n}, \underset{P \times(T-P) \times K}{\tilde{\mathbf{X}}} \text { contains lagged values of } \mathbf{X} \\
& \underset{P \times K}{\mathbf{B}_{n}}=\mathbf{Y}_{\cdot, n}^{\prime} \tilde{\mathbf{X}}+\mathbf{d}_{n} \mathbf{X}, \quad \underset{K \times K \times P}{\mathbf{R}}=\mathbf{X}^{\prime} \tilde{\mathbf{X}}, \underset{P \times K \times P}{\mathbf{D}_{n}}=\mathbf{d}_{n} \tilde{\mathbf{X}} \underset{P \times K \times K \times P}{\mathbf{S}}=\tilde{\mathbf{X}} \tilde{\mathbf{X}}
\end{aligned}
$$

The motivation for using this seemingly cumbersome notation is that it allows for precomputation of all sums and matrix products over the time dimension, so that these can be avoided in each iteration of the algorithm, giving greatly reduced computation times.

\subsection{Conditional log posterior}

Following the derivation in Sidén et al. (2017, Appendix A), we obtain $\boldsymbol{\beta} \mid \mathbf{y}, \boldsymbol{\theta} \sim \mathcal{N}\left(\tilde{\boldsymbol{\mu}}, \tilde{\mathbf{Q}}^{-1}\right)=\mathcal{N}\left(\tilde{\mathbf{Q}}^{-1} \mathbf{b}, \tilde{\mathbf{Q}}^{-1}\right)$ with

$$
\tilde{\mathbf{Q}}=\mathbf{X}^{T} \mathbf{X} \otimes \operatorname{diag}(\boldsymbol{\lambda})+\mathbf{Q}, \quad \tilde{\boldsymbol{\mu}}=\tilde{\mathbf{Q}}^{-1} \operatorname{vec}\left(\operatorname{diag}(\boldsymbol{\lambda}) \mathbf{Y}^{T} \mathbf{X}\right),
$$

for $P=0$ and for the case with autoregressive noise $(P>0)$ we have

$$
\tilde{\mathbf{Q}}=\mathbf{P}_{K N}^{T} \underset{n \in\{1, \ldots, N\}}{\operatorname{blkdiag}}\left[\lambda_{n} \tilde{\mathbf{Q}}_{n}\right] \mathbf{P}_{K N}+\mathbf{Q}, \quad \tilde{\boldsymbol{\mu}}=\tilde{\mathbf{Q}}^{-1} \operatorname{vec}\left(\operatorname{diag}(\boldsymbol{\lambda})\left[\begin{array}{c}
\vdots \\
\tilde{\mathbf{q}}_{n} \\
\vdots
\end{array}\right]_{n \in\{1, \ldots, N\}}\right)
$$

$\tilde{\mathbf{Q}}_{n}=\mathbf{X}^{T} \mathbf{X}-\mathbf{R} \mathbf{A} \cdot, n-(\mathbf{R A} \cdot, n)^{T}+\mathbf{A}_{\cdot, n}^{T} \mathbf{S} \mathbf{A}_{\cdot, n}, \quad \tilde{\mathbf{q}}_{n}=\mathbf{Y}_{\cdot, n}^{T} \mathbf{X}-\mathbf{A}_{\cdot, n}^{T} \mathbf{B}_{n}+\mathbf{A}_{\cdot, n}^{T} \mathbf{D}_{n} \mathbf{A}_{\cdot, n}$

where $\mathbf{P}_{K N}$ is the permutation matrix defined such that $\operatorname{vec}(\mathbf{W})=\mathbf{P}_{K N} \operatorname{vec}\left(\mathbf{W}^{T}\right)$. We note that we can also write the parameters of the i.i.d. case on the second, slightly more complicated form in Eq. (1.3) by instead choosing $\tilde{\mathbf{Q}}_{n}=\mathbf{X}^{T} \mathbf{X}$ and $\tilde{\mathbf{q}}_{n}=\mathbf{Y}_{\cdot, n}^{T} \mathbf{X}$.

\subsection{Gradient}

In this section we derive the gradient for the $\mathrm{M}(2)$ case. The gradient for the other spatial priors can be obtained using the same strategy and these will be left out for brevity. In summary, the log marginal likelihood

$$
\begin{aligned}
\log p(\mathbf{y} \mid \boldsymbol{\theta})= & \frac{T-P}{2} \sum_{n=1}^{N} \log \left(\lambda_{n}\right)-\frac{1}{2} \sum_{n=1}^{N} \lambda_{n} l_{n}\left(\mathbf{W}_{\cdot, n}\right)+\frac{1}{2} \log |\mathbf{Q}|-\frac{1}{2} \boldsymbol{\beta}^{T} \mathbf{Q} \boldsymbol{\beta} \\
& -\frac{1}{2} \log |\tilde{\mathbf{Q}}|+\frac{1}{2}(\boldsymbol{\beta}-\tilde{\boldsymbol{\mu}})^{T} \tilde{\mathbf{Q}}(\boldsymbol{\beta}-\tilde{\boldsymbol{\mu}})+\text { const. }
\end{aligned}
$$


We begin by writing down the log marginal likelihood gradient and then the derivation follows.

$$
\begin{aligned}
& \frac{\partial \log p(\mathbf{y} \mid \boldsymbol{\theta})}{\partial \tau_{k}^{2}}=\frac{N}{2 \tau_{k}^{2}}-\frac{1}{2} \operatorname{tr}\left(\tilde{\mathbf{Q}}^{-1}\left(\mathbf{J}^{k k} \otimes \mathbf{K}_{k} \mathbf{K}_{k}\right)\right)-\frac{1}{2} \mathbf{M}_{k,,} \mathbf{K}_{k} \mathbf{K}_{k} \mathbf{M}_{k, \cdot}^{T}, \\
& \frac{\partial \log p(\mathbf{y} \mid \boldsymbol{\theta})}{\partial \kappa_{k}^{2}}=\operatorname{tr}\left(\mathbf{K}_{k}^{-1}\right)-\tau_{k}^{2} \operatorname{tr}\left(\tilde{\mathbf{Q}}^{-1}\left(\mathbf{J}^{k k} \otimes \mathbf{K}_{k}\right)\right)-\tau_{k}^{2} \mathbf{M}_{k, \cdot} \mathbf{K}_{k} \mathbf{M}_{k, \cdot}^{T}, \\
& \frac{\partial \log p(\mathbf{y} \mid \boldsymbol{\theta})}{\partial \lambda_{n}}=\frac{T-P}{2 \lambda_{n}}-\frac{1}{2} \operatorname{tr}\left(\tilde{\mathbf{Q}}^{-1} \mathbf{P}_{K N}^{T}\left(\mathbf{J}^{n n} \otimes \tilde{\mathbf{Q}}_{n}\right) \mathbf{P}_{K N}\right)-\frac{1}{2} l_{n}\left(\mathbf{M}_{\cdot, n}\right), \\
& \frac{\partial \log p(\mathbf{y} \mid \boldsymbol{\theta})}{\partial \mathbf{A} \cdot, n}=-\left.\frac{1}{2} \lambda_{n} \frac{\partial l_{n}\left(\mathbf{W}_{\cdot, n}\right)}{\partial \mathbf{A}_{\cdot, n}}\right|_{\mathbf{W}=\mathbf{M}}-\frac{1}{2} \frac{\partial \log |\tilde{\mathbf{Q}}|}{\partial \mathbf{A} \cdot, n},
\end{aligned}
$$

where $\mathbf{K}_{k}=\mathbf{G}+\kappa_{k}^{2} \mathbf{I}$ so that $\mathbf{Q}_{k}=\tau_{k}^{2} \mathbf{K}_{k} \mathbf{K}_{k}$ and $\mathbf{J}^{i j}$ is the square single-entry matrix which is zero everywhere except in $(i, j)$ where it is 1 . The size of $\mathbf{J}^{i j}$ is clear from the context and is here used in couple with the Kronecker product to construct singleblock matrices, where everything but one block is zero. $\mathbf{M}$ is the $K \times N$ matrix such that $\tilde{\boldsymbol{\mu}}=\operatorname{vec}\left(\mathbf{M}^{T}\right)$ (compare with $\left.\boldsymbol{\beta}=\operatorname{vec}\left(\mathbf{W}^{T}\right)\right)$. The terms in the expression for $\frac{\partial \log p(\mathbf{y} \mid \boldsymbol{\theta})}{\partial \mathbf{A}_{\cdot, n}}$ are given in Eq. (1.6).

We begin with computing the gradient with respect to $\tau_{k}^{2}$ and $\kappa_{k}^{2}$, that do not appear in the likelihood, which is why the gradient is the same for the case $P=0$ and $P>0$ (given $\tilde{\mathbf{Q}}$ and $\tilde{\boldsymbol{\mu}}$ ). Thereafter, we treat $\lambda_{n}$ which is different in these cases depending on the $\log$ likelihood term expression $l_{n}$ and lastly $A_{p n}$ which is only relevant for the case $P>0$. A reference for some of the matrix algebraic operations used here is Petersen and Pedersen (2012).

\section{Gradient with respect to $\tau_{k}^{2}$ and $\kappa_{k}^{2}$}

Some useful derivatives are

$$
\frac{\partial \mathbf{Q}_{k}}{\partial \tau_{k}^{2}}=\mathbf{K}_{k} \mathbf{K}_{k}, \quad \frac{\partial \mathbf{Q}_{k}}{\partial \kappa_{k}^{2}}=2 \tau_{k}^{2} \mathbf{K}_{k}, \quad \frac{\partial \mathbf{Q}}{\partial \tau_{k}^{2}}=\mathbf{J}^{k k} \otimes \mathbf{K}_{k} \mathbf{K}_{k}, \quad \frac{\partial \mathbf{Q}}{\partial \kappa_{k}^{2}}=\mathbf{J}^{k k} \otimes 2 \tau_{k}^{2} \mathbf{K}_{k} .
$$

Also note that $\frac{\partial \tilde{\mathbf{Q}}}{\partial \tau_{k}^{2}}=\frac{\partial \mathbf{Q}}{\partial \tau_{k}^{2}}$ and $\frac{\partial \tilde{\mathbf{Q}}}{\partial \kappa_{k}^{2}}=\frac{\partial \mathbf{Q}}{\partial \kappa_{k}^{2}}$. Furthermore

$$
\begin{aligned}
\frac{\partial \log \left|\mathbf{Q}_{k}\right|}{\partial \tau_{k}^{2}} & =\frac{\left|\mathbf{Q}_{k}\right|}{\left|\mathbf{Q}_{k}\right|} \operatorname{tr}\left(\mathbf{Q}_{k}^{-1} \frac{\partial \mathbf{Q}_{k}}{\partial \tau_{k}^{2}}\right)=\operatorname{tr}\left(\frac{1}{\tau_{k}^{2}} \mathbf{I}_{N}\right)=\frac{N}{\tau_{k}^{2}}, \quad \frac{\partial \log \left|\mathbf{Q}_{k}\right|}{\partial \kappa_{k}^{2}}=2 \operatorname{tr}\left(\mathbf{K}_{k}^{-1}\right), \\
\frac{\partial \log |\tilde{\mathbf{Q}}|}{\partial \tau_{k}^{2}} & =\operatorname{tr}\left(\tilde{\mathbf{Q}}^{-1}\left(\mathbf{J}^{k k} \otimes \mathbf{K}_{k} \mathbf{K}_{k}\right)\right), \quad \frac{\partial \log |\tilde{\mathbf{Q}}|}{\partial \kappa_{k}^{2}}=\operatorname{tr}\left(\tilde{\mathbf{Q}}^{-1}\left(\mathbf{J}^{k k} \otimes 2 \tau_{k}^{2} \mathbf{K}_{k}\right)\right), \\
\frac{\partial \log \tilde{\boldsymbol{\mu}}^{T} \tilde{\mathbf{Q}} \tilde{\boldsymbol{\mu}}}{\partial \tau_{k}^{2}} & =\mathbf{b}^{T} \frac{\partial \tilde{\mathbf{Q}}^{-1}}{\partial \tau_{k}^{2}} \mathbf{b}=-\mathbf{b}^{T} \tilde{\mathbf{Q}}^{-1} \frac{\partial \tilde{\mathbf{Q}}}{\partial \tau_{k}^{2}} \tilde{\mathbf{Q}}^{-1} \mathbf{b}=-\tilde{\boldsymbol{\mu}}^{T}\left(\mathbf{J}^{k k} \otimes \mathbf{K}_{k} \mathbf{K}_{k}\right) \tilde{\boldsymbol{\mu}} \\
& =-\mathbf{M}_{k,}, \mathbf{K}_{k} \mathbf{K}_{k} \mathbf{M}_{k, \cdot}^{T}, \\
\frac{\partial \log \tilde{\boldsymbol{\mu}}^{T} \tilde{\mathbf{Q}} \tilde{\boldsymbol{\mu}}}{\partial \kappa_{k}^{2}} & =-\tilde{\boldsymbol{\mu}}^{T}\left(\mathbf{J}^{k k} \otimes 2 \tau_{k}^{2} \mathbf{K}_{k}\right) \tilde{\boldsymbol{\mu}}=-2 \tau_{k}^{2} \mathbf{M}_{k, \cdot} \mathbf{K}_{k} \mathbf{M}_{k, \cdot}^{T} .
\end{aligned}
$$


Now, by setting $\boldsymbol{\beta}=\mathbf{0}$ and removing everything that is constant with respect to $\boldsymbol{\tau}^{2}$ and $\kappa^{2}$, Eq. (1.4) becomes

$$
\log p(\mathbf{y} \mid \boldsymbol{\theta})=\frac{1}{2} \sum_{k=1}^{K} \log \left|\mathbf{Q}_{k}\right|-\frac{1}{2} \log |\tilde{\mathbf{Q}}|+\frac{1}{2} \tilde{\boldsymbol{\mu}}^{T} \tilde{\mathbf{Q}} \tilde{\boldsymbol{\mu}}+\text { const }
$$

and we get the derivatives with respect to $\tau_{k}^{2}$ and $\kappa_{k}^{2}$ in Eq. (1.5).

\section{Gradient with respect to $\lambda_{n}$}

Note that

$$
\begin{gathered}
\frac{\partial \tilde{\mathbf{Q}}}{\partial \lambda_{n}^{2}}=\mathbf{P}_{K N}^{T}\left(\mathbf{J}^{n n} \otimes \tilde{\mathbf{Q}}_{n}\right) \mathbf{P}_{K N}, \quad \frac{\partial \log |\tilde{\mathbf{Q}}|}{\partial \lambda_{n}^{2}}=\operatorname{tr}\left(\tilde{\mathbf{Q}}^{-1} \mathbf{P}_{K N}^{T}\left(\mathbf{J}^{n n} \otimes \tilde{\mathbf{Q}}_{n}\right) \mathbf{P}_{K N}\right), \\
\frac{\partial \log (\boldsymbol{\beta}-\tilde{\boldsymbol{\mu}})^{T} \tilde{\mathbf{Q}}(\boldsymbol{\beta}-\tilde{\boldsymbol{\mu}})}{\partial \lambda_{n}^{2}}=(\boldsymbol{\beta}-\tilde{\boldsymbol{\mu}})^{T} \frac{\partial \tilde{\mathbf{Q}}}{\partial \lambda_{n}^{2}}(\boldsymbol{\beta}-\tilde{\boldsymbol{\mu}})-2 \frac{\partial \tilde{\boldsymbol{\mu}}^{T}}{\partial \lambda_{n}^{2}} \tilde{\mathbf{Q}}(\boldsymbol{\beta}-\tilde{\boldsymbol{\mu}}),
\end{gathered}
$$

and that the last expression is zero for $\boldsymbol{\beta}=\tilde{\boldsymbol{\mu}}$. Thus, it is clear that the gradient with respect to $\lambda_{n}$ in Eq. (1.5) can be obtained from taking the derivative of Eq. (1.4) and evaluating at $\boldsymbol{\beta}=\tilde{\boldsymbol{\mu}}$.

\section{Gradient with respect to $A_{p n}$}

Use that

$$
\begin{aligned}
\frac{\partial l_{n}\left(\mathbf{W}_{\cdot, n}\right)}{\partial \mathbf{A}_{\cdot, n}}= & 2\left[-\mathbf{Y}_{\cdot, n}^{T} \mathbf{d}_{n}^{T}+\mathbf{W B}_{n}^{T}-\mathbf{W}_{\cdot, n}^{T} \mathbf{R W} \cdot, n\right. \\
& \left.+\left(\mathbf{d}_{n} \mathbf{d}_{n}^{T}-\mathbf{D}_{n} \mathbf{W}_{\cdot, n}-\left(\mathbf{D}_{n} \mathbf{W}_{\cdot, n}\right)^{T}+\mathbf{W}_{\cdot, n}^{T} \mathbf{S} \mathbf{W}_{\cdot, n}\right) \mathbf{A}_{\cdot, n}\right], \\
\frac{\partial \tilde{\mathbf{Q}}_{n}}{\partial A_{p, n}}= & -\mathbf{R}_{p}-\mathbf{R}_{p}^{T}+\mathbf{S}_{p} \mathbf{A}_{\cdot, n}+\left(\mathbf{S}_{p} \mathbf{A}_{\cdot, n}\right)^{T}, \\
\frac{\partial \log |\tilde{\mathbf{Q}}|}{\partial A_{p, n}}= & \operatorname{tr}\left(\tilde{\mathbf{Q}}^{-1} \mathbf{P}_{K N}^{T}\left(\mathbf{J}^{n n} \otimes \lambda_{n} \frac{\partial \tilde{\mathbf{Q}}_{n}}{\partial A_{p, n}}\right) \mathbf{P}_{K N}\right),
\end{aligned}
$$

where $\mathbf{R}_{p}$ and $\mathbf{S}_{p}$ are of sizes $K \times K$ and $K \times K \times P$ and refers to the $p$ th sub-tensor from the appropriate dimension of $\mathbf{R}$ and $\mathbf{S}$ respectively. The expression in Eq. (1.4) is derived after noting that the remaining terms of the log likelihood become zero after taking the derivative and evaluating at $\boldsymbol{\beta}=\tilde{\boldsymbol{\mu}}$. 


\subsection{Approximate Hessian}

The approximate Hessian for the log marginal likelihood in the $\mathrm{M}(2)$ case, computed directly with respect to the parameters $\tau_{0, k}=\log \left(\tau_{k}^{2}\right), \kappa_{0, k}=\log \left(\kappa_{k}^{2}\right)$ is

$$
\begin{aligned}
E_{\boldsymbol{\beta} \mid \mathbf{Y}, \boldsymbol{\theta}}\left[\frac{\partial^{2} \log p(\mathbf{y}, \boldsymbol{\beta} \mid \boldsymbol{\theta})}{\partial \tau_{0, k}^{2}}\right]= & -\frac{\tau_{k}^{2}}{2} \operatorname{tr}\left(\tilde{\mathbf{Q}}^{-1}\left(\mathbf{J}^{k k} \otimes \mathbf{K}_{k} \mathbf{K}_{k}\right)\right)-\frac{1}{2} \mathbf{M}_{k, \cdot} \mathbf{Q}_{k} \mathbf{M}_{k, \cdot}^{T}, \\
E_{\boldsymbol{\beta} \mid \mathbf{Y}, \boldsymbol{\theta}}\left[\frac{\partial^{2} \log p(\mathbf{y}, \boldsymbol{\beta} \mid \boldsymbol{\theta})}{\partial \kappa_{0, k}^{2}}\right]= & \kappa_{k}^{2}\left[\operatorname{tr}\left(\mathbf{K}_{k}^{-1}\right)-\kappa_{k}^{2} \tau_{k}^{2} \operatorname{tr}\left(\mathbf{K}_{k}^{-1} \mathbf{K}_{k}^{-1}\right)+\right. \\
& -\tau_{k}^{2} \mathbf{M}_{k, \cdot} \mathbf{K}_{k} \mathbf{M}_{k, \cdot}^{T}-\tau_{k}^{2} \operatorname{tr}\left(\tilde{\mathbf{Q}}^{-1}\left(\mathbf{J}^{k k} \otimes \mathbf{K}_{k}\right)\right)+ \\
& -\kappa_{k}^{2} \tau_{k}^{2} \mathbf{M}_{k, \cdot} \mathbf{M}_{k, \cdot}^{T}-\kappa_{k}^{2} \tau_{k}^{2} \operatorname{tr}\left(\tilde{\mathbf{Q}}^{-1}\left(\mathbf{J}^{k k} \otimes \mathbf{I}\right)\right) .
\end{aligned}
$$

The derivation starts by noting that the non-constant part of the augmented log likelihood with respect to $\tau_{0, k}$ and $\kappa_{0, k}$ is

$$
\log p(\mathbf{y}, \boldsymbol{\beta} \mid \boldsymbol{\theta})=\frac{1}{2} \log |\mathbf{Q}|-\frac{1}{2} \boldsymbol{\beta}^{T} \mathbf{Q} \boldsymbol{\beta}+\text { const. }
$$

Taking the derivative twice with respect to $\tau_{0, k}$ and $\kappa_{0, k}$ and computing the expectation gives the result, after noting that $E_{\boldsymbol{\beta} \mid \mathbf{Y}, \boldsymbol{\theta}}\left[\boldsymbol{\beta}^{T} \mathbf{T} \boldsymbol{\beta}\right]=\operatorname{tr}\left(\tilde{\mathbf{Q}}^{-1} \mathbf{T}\right)+\tilde{\boldsymbol{\mu}}^{T} \mathbf{T} \tilde{\boldsymbol{\mu}}$, for general $K N \times K N$ matrix $\mathbf{T}$ (Petersen and Pedersen, 2012, Eq. (318)). We write out the derivation for $\tau_{0, k}$ as an example. First note that

$$
\frac{\partial \mathbf{Q}}{\partial \tau_{0, k}}=\mathbf{J}^{k k} \otimes \frac{\partial}{\partial \tau_{0, k}} \exp \left(\tau_{0, k}\right) \mathbf{K}_{k} \mathbf{K}_{k}=\mathbf{J}^{k k} \otimes \mathbf{Q}_{k},
$$

so

$$
\begin{aligned}
\frac{\partial \log p(\mathbf{y}, \boldsymbol{\beta} \mid \boldsymbol{\theta})}{\partial \tau_{0, k}} & =\frac{1}{2} \operatorname{tr}\left(\mathbf{Q}_{k}^{-1} \mathbf{Q}_{k}\right)-\frac{1}{2} \mathbf{W}_{k, \cdot} \mathbf{Q}_{k} \mathbf{W}_{k, \cdot}^{T}=\frac{N}{2}-\frac{1}{2} \mathbf{W}_{k, \cdot} \mathbf{Q}_{k} \mathbf{W}_{k, \cdot}^{T}, \\
\frac{\partial^{2} \log p(\mathbf{y}, \boldsymbol{\beta} \mid \boldsymbol{\theta})}{\partial \tau_{0, k}^{2}} & =-\frac{1}{2} \mathbf{W}_{k, \cdot} \mathbf{Q}_{k} \mathbf{W}_{k,}^{T},
\end{aligned}
$$

and taking the conditional expectation with respect to $\boldsymbol{\beta} \mid \mathbf{Y}, \boldsymbol{\theta}$ gives the result in Eq. (1.7).

\subsection{The anisotropic case}

We will in this subsection present the gradient and Hessian for the anisotropic $\mathrm{M}(2)$ model. We only consider $h_{0, k, x}=\log \left(h_{k, x}\right)$, as the results for $h_{k, y}$ are completely symmetric. The derivations can be performed analogously to Subsection 1.3 and Subsection 1.4. The gradient is

$$
\begin{aligned}
\frac{\partial \log p(\mathbf{y} \mid \boldsymbol{\theta})}{\partial h_{0, k, x}}= & \operatorname{tr}\left(\mathbf{K}_{k}^{-1} \frac{\partial \mathbf{K}_{k}}{\partial h_{0, k, x}}\right)-\tau_{k}^{2} \operatorname{tr}\left(\tilde{\mathbf{Q}}^{-1}\left(\mathbf{J}^{k k} \otimes\left(\mathbf{K}_{k} \frac{\partial \mathbf{K}_{k}}{\partial h_{0, k, x}}\right)\right)\right)+ \\
& -\tau_{k}^{2} \mathbf{M}_{k,}, \mathbf{K}_{k} \frac{\partial \mathbf{K}_{k}}{\partial h_{0, k, x}} \mathbf{M}_{k,,}^{T}
\end{aligned}
$$


where $\frac{\partial \mathbf{K}_{k}}{\partial h_{0, k, x}}=\exp \left(h_{0, k, x}\right) \mathbf{G}_{x}-\exp \left(-h_{0, k, x}\right) \exp \left(-h_{0, k, y}\right) \mathbf{G}_{z}$. The approximate Hessian is

$$
\begin{aligned}
E_{\boldsymbol{\beta} \mid \mathbf{Y}, \boldsymbol{\theta}}\left[\frac{\partial^{2} \log p(\mathbf{y}, \boldsymbol{\beta} \mid \boldsymbol{\theta})}{\partial h_{0, k, x}^{2}}\right]= & -\operatorname{tr}\left(\mathbf{K}_{k}^{-1} \frac{\partial \mathbf{K}_{k}}{\partial h_{0, k, x}} \mathbf{K}_{k}^{-1} \frac{\partial \mathbf{K}_{k}}{\partial h_{0, k, x}}\right)+\operatorname{tr}\left(\mathbf{K}_{k}^{-1} \frac{\partial^{2} \mathbf{K}_{k}}{\partial h_{0, k, x}^{2}}\right)+ \\
& -\tau_{k}^{2}\left[\mathbf{M}_{k, \cdot} \mathbf{H}_{k, x} \mathbf{M}_{k, \cdot}^{T}+\operatorname{tr}\left(\tilde{\mathbf{Q}}^{-1}\left(\mathbf{J}^{k k} \otimes \mathbf{H}_{k, x}\right)\right)\right]
\end{aligned}
$$

where $\frac{\partial^{2} \mathbf{K}_{k}}{\partial h_{0, k, x}^{2}}=\exp \left(h_{0, k, x}\right) \mathbf{G}_{x}+\exp \left(-h_{0, k, x}\right) \exp \left(-h_{0, k, y}\right) \mathbf{G}_{z}$ and

$\mathbf{H}_{k, x}=\frac{\partial \mathbf{K}_{k}}{\partial h_{0, k, x}} \frac{\partial \mathbf{K}_{k}}{\partial h_{0, k, x}}+\mathbf{K}_{k} \frac{\partial^{2} \mathbf{K}_{k}}{\partial h_{0, k, x}^{2}}$. The first trace of this expression will be Hutchinson approximated using $\operatorname{tr}\left(\mathbf{K}_{k}^{-1} \frac{\partial \mathbf{K}_{k}}{\partial h_{0, k, x}} \mathbf{K}_{k}^{-1} \frac{\partial \mathbf{K}_{k}}{\partial h_{0, k, x}}\right) \approx \frac{1}{N_{s}} \sum_{j=1}^{N_{s}} \mathbf{v}_{j}^{T} \mathbf{K}_{k}^{-1} \frac{\partial \mathbf{K}_{k}}{\partial h_{0, k, x}} \mathbf{K}_{k}^{-1} \frac{\partial \mathbf{K}_{k}}{\partial h_{0, k, x}} \mathbf{v}_{j}$, which requires solving two equation systems for every term and a somewhat higher computational cost.

\subsection{Spatial hyperparameter priors}

This section covers the priors of the spatial hyperparameters and their derivatives and second derivatives. We drop the sub-indexing with respect to $k$ throughout this section as the parameter priors are mutually independent.

\section{Priors for $\tau^{2}$ and $\kappa^{2}$ for $\mathrm{M}(2)$}

For the spatial Matérn prior with $\alpha=2$ the joint PC $\log$ prior for $\tau^{2}$ and $\kappa$ is

$$
\log p\left(\tau^{2}, \kappa\right)=-\frac{3}{2} \log \tau^{2}+\left(\frac{d}{2}-1-\nu\right) \log \kappa-\lambda_{1} \kappa^{d / 2}-\lambda_{3} \kappa^{-\nu}\left(\tau^{2}\right)^{-1 / 2}+\text { const. }
$$

The PC prior controls the spatial range $\rho$ and marginal variance $\sigma^{2}$ of the spatial field, which are a bijective transform of $\tau^{2}$ and $\kappa$, through a priori probabilities $P\left(\rho<\rho_{0}\right)=$ $\xi_{1}$ and $P\left(\sigma^{2}>\sigma_{0}^{2}\right)=\xi_{2}$. This generates the constants $\lambda_{1}=-\log \left(\xi_{1}\right)\left(\rho_{0} / \sqrt{8 \nu}\right)^{d / 2}$ and $\lambda_{3}=-\frac{\log \left(\xi_{2}\right)}{\sigma_{0}} \sqrt{\frac{\Gamma(\nu)}{\Gamma(\nu+d / 2)(4 \pi)^{d / 2}}}$ in Eq. (1.10). As default we will use the values $\xi_{1}=\xi_{2}=0.05, \rho_{0}=2$ voxels and $\sigma_{0}$ corresponding to $2 \%$ of the global mean signal. It is straightforward to obtain the derivatives

$$
\begin{aligned}
& \frac{\partial \log p\left(\tau^{2}, \kappa\right)}{\partial\left(\tau^{2}\right)}=-\frac{3}{2 \tau^{2}}+\frac{\lambda_{3} \kappa^{-\nu}}{2}\left(\tau^{2}\right)^{-3 / 2} \\
& \frac{\partial \log p\left(\tau^{2}, \kappa\right)}{\partial\left(\kappa^{2}\right)}=\frac{1}{2 \kappa}\left(\frac{d / 2-1-\nu}{\kappa}-\frac{\lambda_{1} d}{2} \kappa^{d / 2-1}+\lambda_{3} \nu \kappa^{-\nu-1}\left(\tau^{2}\right)^{-1 / 2}\right) .
\end{aligned}
$$

Changing parameterization to $\tau_{0}$ and $\kappa_{0}$ as before and taking the second derivative with respect to these gives

$$
\frac{\partial^{2} \log p\left(\tau_{0}, \kappa_{0}\right)}{\partial \tau_{0}^{2}}=-\frac{\lambda_{3}}{4} \exp \left(-\frac{\nu \kappa_{0}}{2}\right) \exp \left(-\frac{\tau_{0}}{2}\right)
$$




$$
\begin{aligned}
\frac{\partial^{2} \log p\left(\tau_{0}, \kappa_{0}\right)}{\partial \kappa_{0}^{2}}=\frac{1}{4} & {\left[-(-d / 2-1-\nu) \exp \left(-\frac{\kappa_{0}}{2}\right)-\left(\frac{d}{2}-1\right) \frac{\lambda_{1} d}{2} \exp \left(\frac{\kappa_{0}}{2}\left(\frac{d}{2}-1\right)\right)\right.} \\
& \left.+(\nu-1) \lambda_{3} \nu \exp \left(\frac{\kappa_{0}}{2}(\nu-1)\right) \exp \left(-\frac{\tau_{0}}{2}\right)\right] .
\end{aligned}
$$

Priors for $\tau^{2}$ and $\kappa^{2}$ for $\mathrm{M}(1)$

In this situation, we use independent log-normal priors for $\tau^{2}$ and $\kappa^{2}$, that is $\tau_{0} \sim$ $\mathcal{N}\left(\mu_{\tau_{0}}, \sigma_{\tau_{0}}^{2}\right)$ and $\kappa_{0} \sim \mathcal{N}\left(\mu_{\kappa_{0}}, \sigma_{\kappa_{0}}^{2}\right)$. For $\tau_{0}$ we have the derivatives

$$
\frac{\partial \log p\left(\tau^{2}, \kappa\right)}{\partial \tau_{0}}=-\frac{\tau_{0}-\mu_{\tau_{0}}}{\sigma_{\tau_{0}}^{2}}, \quad \frac{\partial^{2} \log p\left(\tau^{2}, \kappa\right)}{\partial \tau_{0}^{2}}=-\frac{1}{\sigma_{\tau_{0}}^{2}},
$$

and correspondingly for $\kappa_{0}$. Per default we use $\mu_{\tau_{0}}=\log 0.01, \mu_{\kappa_{0}}=\log 0.1, \sigma_{\tau_{0}}=4$, $\sigma_{\kappa_{0}}=1$.

Priors for $h_{x}$ and $h_{y}$ for the anisotropic prior

We use a log-normal prior for $h_{x}$ and $h_{y}$. For $h_{0, x}=\log \left(h_{x}\right)$ we have the derivatives

$$
\frac{\partial \log p\left(h_{0, x}, h_{0, y}\right)}{\partial h_{0, x}}=-\frac{2}{3 \sigma_{h}^{2}}\left(h_{0, x}-\frac{h_{0, y}}{2}\right), \quad \frac{\partial^{2} \log p\left(h_{0, x}, h_{0, y}\right)}{\partial h_{0, x}^{2}}=-\frac{2}{3 \sigma_{h}^{2}},
$$

and correspondingly for $h_{0, y}$.

\section{Priors for $\tau^{2}$ for $\operatorname{ICAR}(1)$ and $\operatorname{ICAR}(2)$}

We use the PC prior for $\tau^{2}$ for Gaussian random effects from Simpson et al. (2017)

$$
p\left(\tau^{2}\right)=\frac{\lambda_{2}}{2}\left(\tau^{2}\right)^{-3 / 2} \exp \left(-\lambda_{2}\left(\tau^{2}\right)^{-1 / 2}\right), \quad \tau^{2}>0
$$

By specifying $\sigma_{0}^{2}$ and $\xi_{2}$ so that $P\left(\sigma_{i \mid-i}^{2}>\sigma_{0}^{2}\right)=\xi_{2}$, we get $\lambda_{2}=-\log \left(\xi_{2}\right) /\left(\sigma_{0} \sqrt{6}\right)$ for $\operatorname{ICAR}(1)$ and $\lambda_{2}=-\log \left(\xi_{2}\right) /\left(\sigma_{0} \sqrt{42}\right)$ for $\operatorname{ICAR}(2)$. Derivatives are obtained as

$$
\begin{gathered}
\frac{\partial \log p\left(\tau^{2}\right)}{\partial\left(\tau^{2}\right)}=-\frac{3}{2 \tau^{2}}+\frac{\lambda_{2}}{2}\left(\tau^{2}\right)^{-3 / 2} \\
\frac{\partial^{2} \log p\left(\tau_{0}\right)}{\partial \tau_{0}^{2}}=-\frac{\lambda_{2}}{4} \exp \left(-\frac{\tau_{0}}{2}\right) .
\end{gathered}
$$

When comparing to results from our older paper (Sidén et al., 2017), we use the same gamma prior as used there for $\operatorname{ICAR}(1), \tau^{2} \sim \Gamma\left(q_{1}, q_{2}\right)$, which has derivatives

$$
\begin{array}{r}
\frac{\partial \log p\left(\tau^{2}\right)}{\partial\left(\tau^{2}\right)}=q_{2}-1-\frac{\tau^{2}}{q_{1}} \\
\frac{\partial^{2} \log p\left(\tau_{0}\right)}{\partial \tau_{0}^{2}}=-\frac{1}{q_{1}} \exp \left(\tau_{0}\right) .
\end{array}
$$

We use the default values $q_{1}=10$ and $q_{2}=0.1$. 


\section{Cross-Validation}

\subsection{Cross-validation over left out voxels}

To reduce the impact of the noise model, we drop the entire BOLD time series for $d \%$ of the voxels to obtain the cross-validation voxel set $D$, and predict the time series $\mathbf{Y}_{\cdot, D}$ given the other data $\mathbf{Y}_{\cdot, D^{c}}$. To separate the activation and nuisance regressors, we rewrite the model as

$$
\underset{T \times N}{\mathbf{Y}}=\underset{T \times K K \times N}{\mathbf{X}} \underset{\mathbf{W}}{\mathbf{W}}+\mathbf{E}=\left[\begin{array}{cc}
\dot{\mathbf{X}} & \ddot{\mathbf{X}}
\end{array}\right]\left[\begin{array}{c}
\dot{\mathbf{W}} \\
\ddot{\mathbf{W}}
\end{array}\right]+\mathbf{E}=\dot{\mathbf{X}} \dot{\mathbf{W}}+\ddot{\mathbf{X}} \ddot{\mathbf{W}}+\mathbf{E},
$$

with $\dot{\mathbf{W}}$ corresponding to the $K_{a c t} \times N$ activity regression coefficients, which have spatial priors, and $\ddot{\mathbf{W}}$ corresponding to the nuisance regression coefficients. We define the cross-validation error time series as

$$
\begin{aligned}
& \mathbf{E}_{\cdot, D}^{C V}=\mathbf{R}_{\cdot, D}-\ddot{\mathbf{X}} E\left(\ddot{\mathbf{W}}_{\cdot, D} \mid \mathbf{R}_{\cdot, D}, \boldsymbol{\theta}\right), \\
& \mathbf{R}_{\cdot, D}=\mathbf{Y}_{\cdot, D}-\dot{\mathbf{X}} E\left(\dot{\mathbf{W}}_{\cdot, D} \mid \mathbf{Y}_{\cdot,-D}, \boldsymbol{\theta}\right),
\end{aligned}
$$

which is computed in two steps. First the out-of-sample residuals $\mathbf{R}_{, D}$ of the spatial part of the model are computed and then a new model $\mathbf{R}_{\cdot, D}=\ddot{\mathbf{X}} \ddot{\mathbf{W}}_{\cdot, D}+\mathbf{E}_{\cdot, D}$ is fitted for each voxel independently, using the original values for the parameters $\boldsymbol{\theta}$, before the error time series $\mathbf{E}_{\cdot . D}^{C V}$ can be computed. The reason for this seemingly complicated procedure is to reduce the impact of the nuisance regressors on the evaluation of the spatial model. We compute the in-sample errors as $\mathbf{E}^{I S}=\mathbf{Y}-\mathbf{X} E(\mathbf{W} \mid \mathbf{Y}, \boldsymbol{\theta})$. We compute the MAE and RMSE for voxel set $D$ as

$$
\mathrm{MAE}=\frac{1}{T|D|} \sum_{t=1}^{T} \sum_{n \in D}\left|\mathbf{E}_{t, n}\right|, \quad \mathrm{RMSE}=\sqrt{\frac{1}{T|D|} \sum_{t=1}^{T} \sum_{n \in D} \mathbf{E}_{t, n}^{2} .}
$$

The spatial posterior predictions for the dropped voxels $E\left(\dot{\mathbf{W}}_{\cdot, D} \mid \mathbf{Y}_{\cdot,-D}, \boldsymbol{\theta}\right)$ are computed using Eq. (1.3) after replacing $\tilde{\mathbf{Q}}_{n}$ and $\tilde{\mathbf{q}}_{n}$ with $\mathbf{0}$ for all $n \in D$.

For the proper scoring rules CRPS, IGN and INT, we also need to compute the predictive standard deviation in each voxel. This is done in a way that neglects the uncertainty in the intercept and head motion regressors. For an unseen datapoint $\tilde{\mathbf{Y}}_{t, n}$, the law of total variance gives

$$
\begin{aligned}
\operatorname{Var}\left(\tilde{\mathbf{Y}}_{t, n} \mid \mathbf{Y}_{\cdot,-D}, \boldsymbol{\theta}, \ddot{\mathbf{W}}\right) & =\mathrm{E}_{\dot{\mathbf{W}} \mid \mathbf{Y}_{\cdot,-D}}\left[\operatorname{Var}\left(\tilde{\mathbf{Y}}_{t, n} \mid \boldsymbol{\theta}, \mathbf{W}\right)\right]+\operatorname{Var}_{\dot{\mathbf{W}} \mid \mathbf{Y} \cdot,-D}\left[\mathrm{E}\left(\tilde{\mathbf{Y}}_{t, n} \mid \boldsymbol{\theta}, \mathbf{W}\right)\right] \\
& =\operatorname{Var}\left(\tilde{\mathbf{Y}}_{t, n} \mid \boldsymbol{\theta}, \mathbf{W}\right)+\mathbf{X}_{t,\left(1: K_{a c t}\right)} \operatorname{Var}\left(\dot{\mathbf{W}} \mid \mathbf{Y}_{\cdot,-D}\right) \mathbf{X}_{t,\left(1: K_{a c t}\right)}^{T}
\end{aligned}
$$

where the first term is simply the variance of the AR noise process in voxel $n$ that does not depend on $\mathbf{W}$ and which can be obtained given the AR parameters $\mathbf{A}_{\cdot, n}$ through the 
Yule-Walker equations (see for example Cryer and Chan, 2008). The second term can be computed using the simple RBMC estimator as in Eq. (3.2) in the main article after replacing $\tilde{\mathbf{Q}}_{n}$ with $\mathbf{0}$ for all $n \in D$ as was done for the mean. The predictive distribution for $x=\mathbf{E}_{t, n}$ is Gaussian with mean $\mu=0$ and variance $\sigma^{2}$ as in Eq. (2.4) which gives simple expressions for the scores as

$$
\begin{aligned}
\mathrm{CRPS}_{t, n} & =\sigma\left[\frac{1}{\sqrt{\pi}}-2 \varphi\left(\frac{x-\mu}{\sigma}\right)-\frac{x-\mu}{\sigma}\left(2 \Phi\left(\frac{x-\mu}{\sigma}\right)-1\right)\right], \\
\mathrm{IGN}_{t, n} & =\log \left(\frac{1}{\sigma} \varphi\left(\frac{x-\mu}{\sigma}\right)\right), \\
\mathrm{INT}_{t, n} & =2 A \sigma+\frac{2}{u}[(\mu-A \sigma-x) \mathbf{1}(x<\mu-A \sigma)+(x-(\mu+A \sigma)) \mathbf{1}(x>\mu+A \sigma)],
\end{aligned}
$$

where $\varphi$ and $\Phi$ denotes the standard normal PDF and CDF and $A=\Phi^{-1}(1-u / 2) \approx$ 1.96 for $u=0.05$, which is used by default. The presented values for the scores are averages across all time points and left out voxels.

\section{Additional Results}
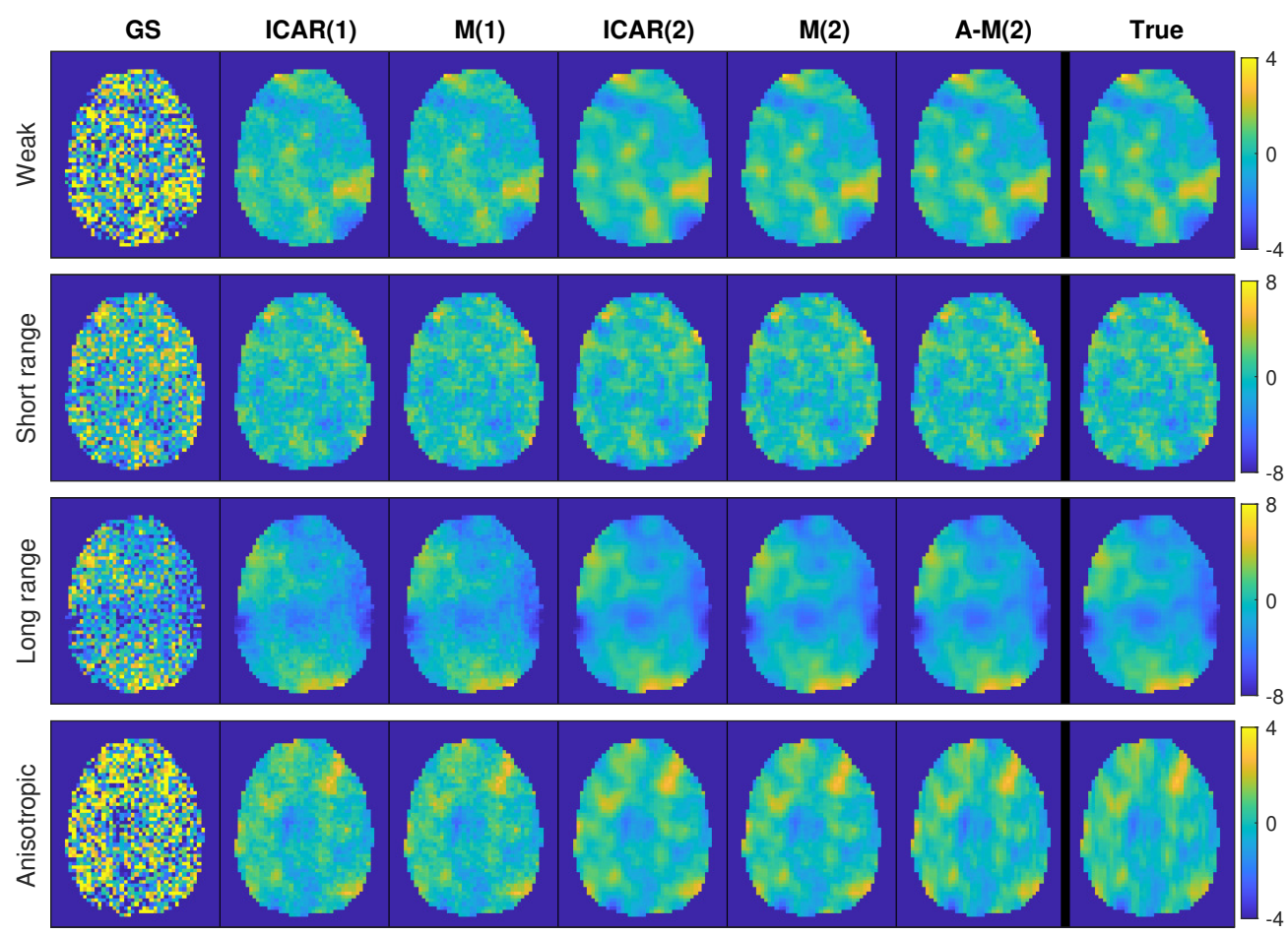

Figure 1: Posterior means of activity coefficients for the four conditions of the simulated dataset, estimated with different spatial priors, which are used in computation of the PPMs in Fig. 2. 
10
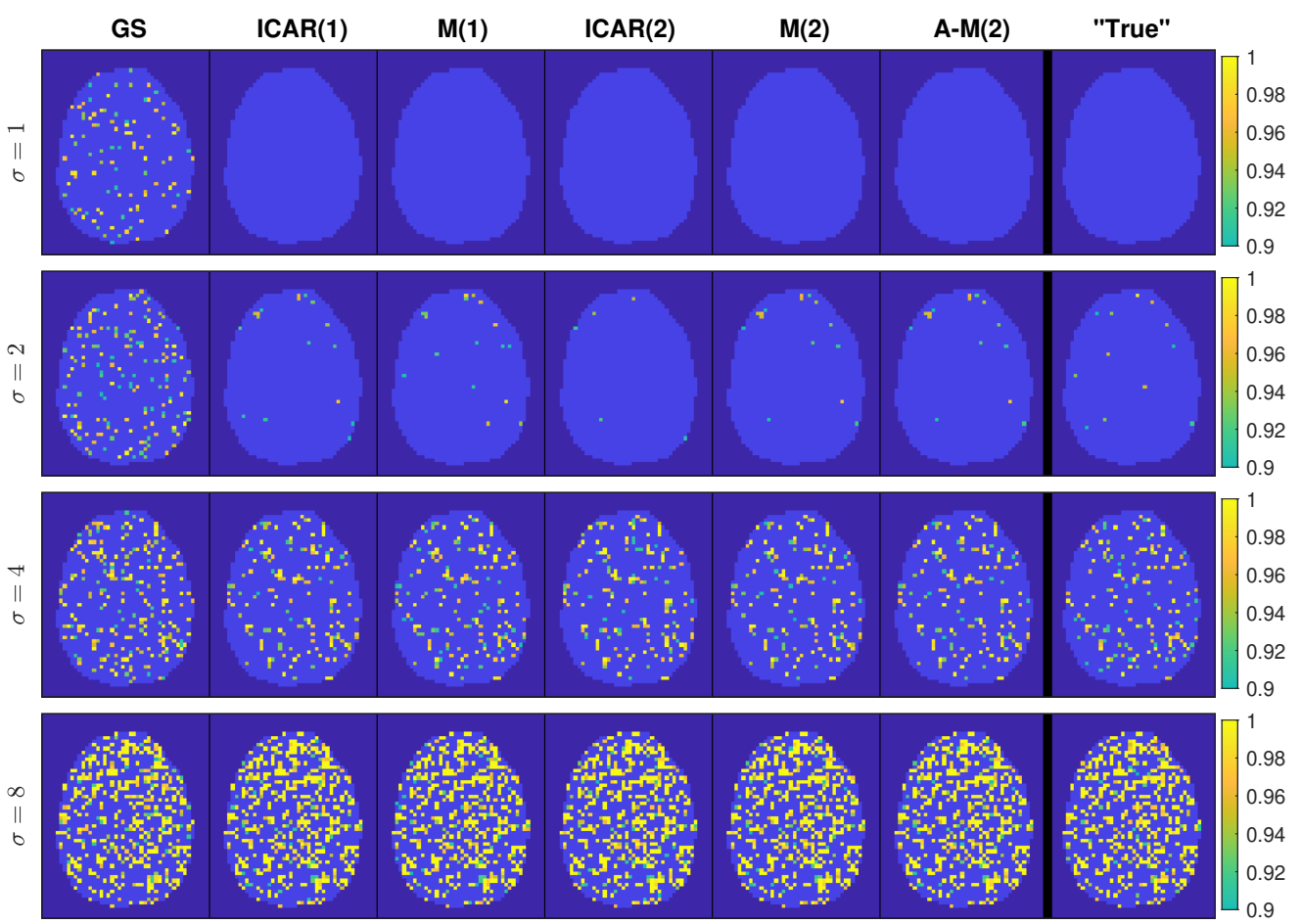

Figure 2: PPMs for the four conditions of the additional simulated dataset with spatially independent activations, estimated with different spatial priors. The last column "True" shows the results when the true spatially independent model used to generate the data is used for estimation. The PPMs show probabilities of exceeding $0.2 \%$ of the global mean signal, thresholded at 0.9 . 


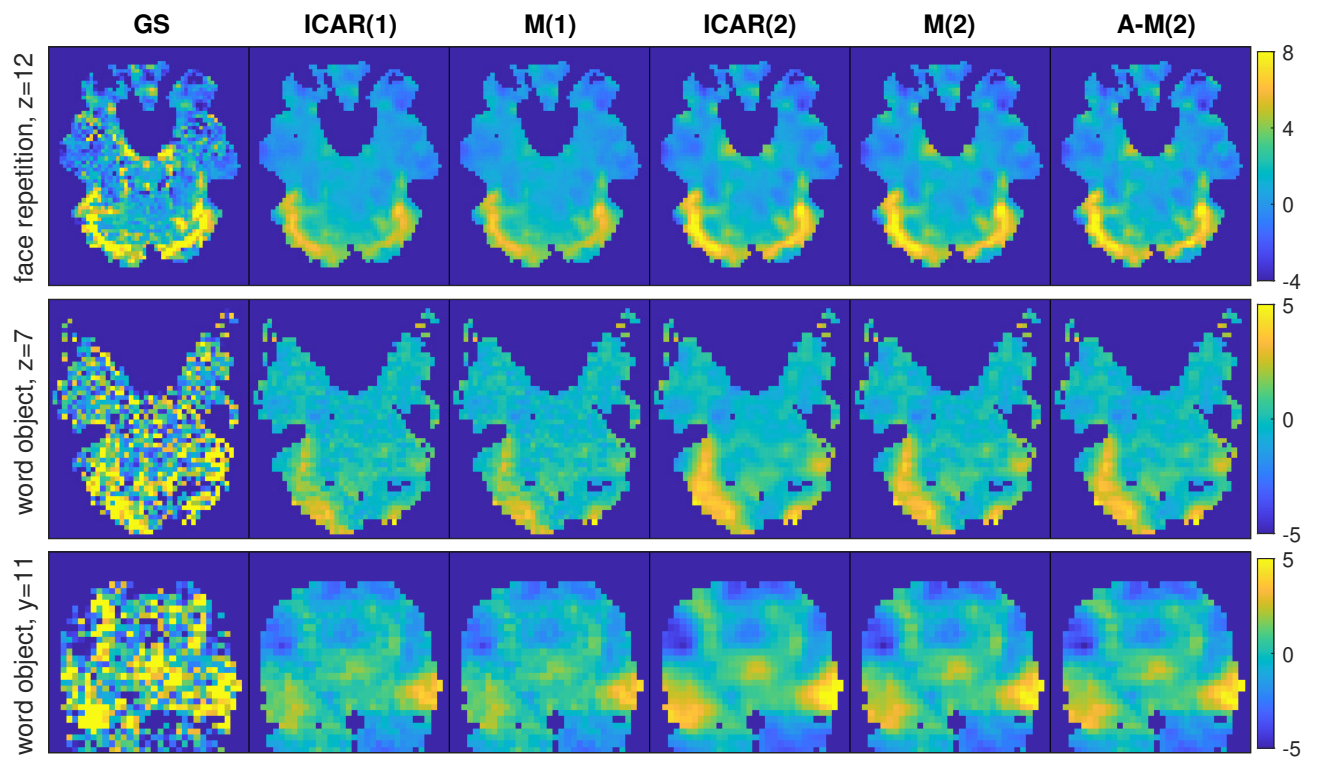

Figure 3: Posterior means for the two experimental datasets, when using different spatial priors, which are used in computation of the PPMs in Fig. 3. The top row shows axial slice 12 of the face repetition dataset, and the middle and bottom rows show axial slice 7 and coronal slice 11 of the word object dataset. 

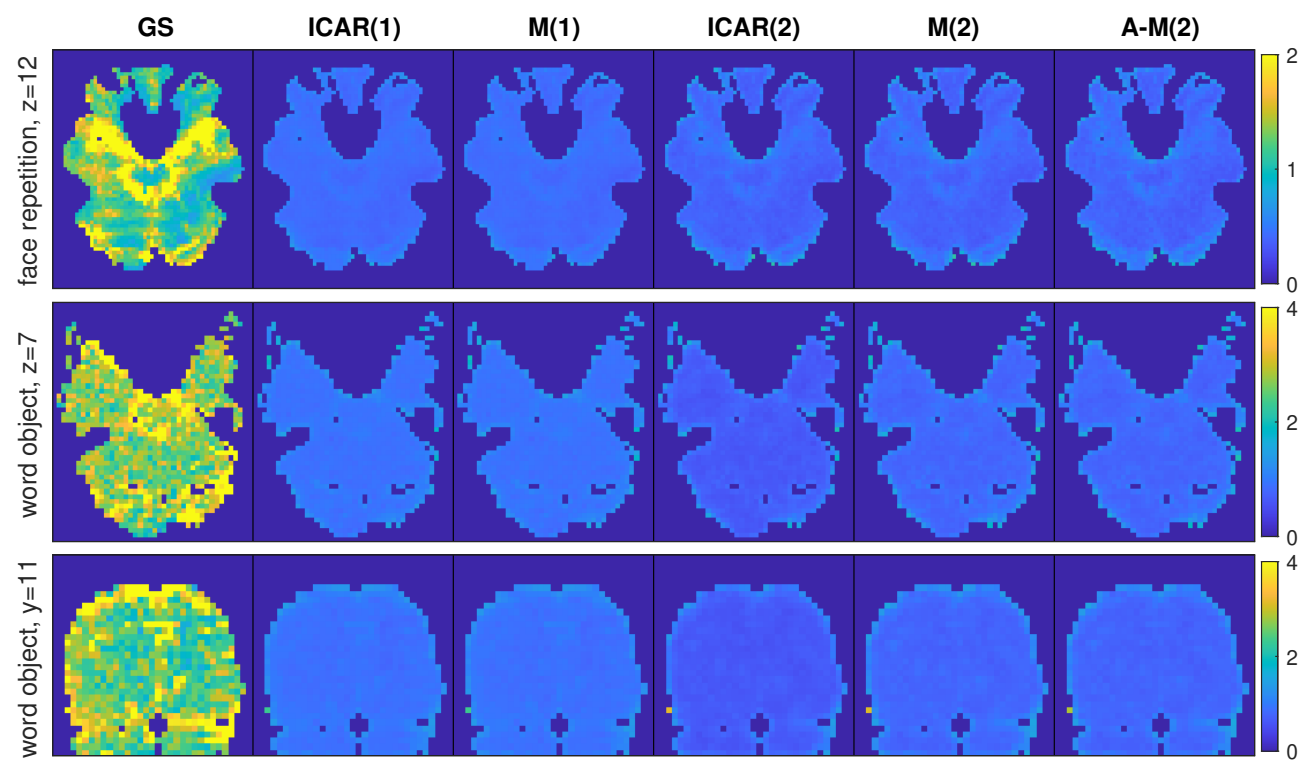

Figure 4: Posterior standard deviations for the two experimental datasets, when using different spatial priors, which are used in computation of the PPMs in Fig. 3. The top row shows axial slice 12 of the face repetition dataset, and the middle and bottom rows show axial slice 7 and coronal slice 11 of the word object dataset. 
Table 1: Cross-validation scores for the two datasets, comparing the different spatial priors. The scores are computed as means across voxels, and presented in negatively oriented forms, so that smaller values are always better. In-sample refers to the average across all voxels, while the other columns shows means and standard errors across 50 random sets of left out voxels.

\begin{tabular}{|c|c|c|c|c|c|c|c|}
\hline \multirow[b]{2}{*}{ Prior } & \multirow[b]{2}{*}{ Score } & \multicolumn{3}{|c|}{ Face repetition data } & \multicolumn{3}{|c|}{ Word object data } \\
\hline & & In-sample & $50 \%$ left out & $90 \%$ left out & In-sample & $50 \%$ left out & $90 \%$ left out \\
\hline $\operatorname{ICAR}(1)$ & \multirow{5}{*}{ MAE } & 1.4948 & $1.5032( \pm 0.0005)$ & $1.5083( \pm 0.0001)$ & 4.7794 & $4.8243( \pm 0.0012)$ & $4.8293( \pm 0.0003)$ \\
\hline $\mathrm{M}(1)$ & & 1.4940 & $1.5026( \pm 0.0006)$ & $1.5074( \pm 0.0001)$ & 4.7792 & $4.8244( \pm 0.0012)$ & $4.8293( \pm 0.0003)$ \\
\hline $\operatorname{ICAR}(2)$ & & 1.4936 & $1.5003( \pm 0.0005)$ & $1.5060( \pm 0.0001)$ & 4.7924 & $4.8230( \pm 0.0012)$ & $4.8299( \pm 0.0004)$ \\
\hline $\mathrm{M}(2)$ & & 1.4927 & $1.4994( \pm 0.0005)$ & $1.5051( \pm 0.0001)$ & 4.7837 & $4.8226( \pm 0.0012)$ & $4.8283( \pm 0.0003)$ \\
\hline $\mathrm{A}-\mathrm{M}(2)$ & & 1.4918 & $1.4987( \pm 0.0005)$ & $\mathbf{1 . 5 0 4 9}( \pm 0.0001)$ & 4.7842 & 4.8223 $( \pm 0.0012)$ & 4.8282( $( \pm 0.0003)$ \\
\hline $\operatorname{ICAR}(1)$ & \multirow{5}{*}{ RMSE } & 2.0513 & $2.0602( \pm 0.0024)$ & $2.0681( \pm 0.0007)$ & 6.3393 & $6.4007( \pm 0.0039)$ & $6.4018( \pm 0.0011)$ \\
\hline $\mathrm{M}(1)$ & & 2.0545 & $2.0644( \pm 0.0040)$ & $2.0685( \pm 0.0014)$ & 6.3391 & $6.4007( \pm 0.0039)$ & $6.4019( \pm 0.0011)$ \\
\hline $\operatorname{ICAR}(2)$ & & 2.0573 & $2.0660( \pm 0.0027)$ & $2.0729( \pm 0.0010)$ & 6.3531 & $6.3976( \pm 0.0039)$ & $6.4011( \pm 0.0013)$ \\
\hline$M(2)$ & & 2.0378 & $2.0459( \pm 0.0016)$ & $2.0527( \pm 0.0006)$ & 6.3430 & $6.3975( \pm 0.0040)$ & $6.3998( \pm 0.0011)$ \\
\hline $\mathrm{A}-\mathrm{M}(2)$ & & 2.0317 & $\mathbf{2 . 0 3 9 7}( \pm 0.0015)$ & $\mathbf{2 . 0 4 7 9}( \pm 0.0004)$ & 6.3434 & $\mathbf{6 . 3 9 7 0}( \pm 0.0040)$ & $\mathbf{6 . 3 9 9 7}( \pm 0.0011)$ \\
\hline $\operatorname{ICAR}(1)$ & \multirow{5}{*}{-CRPS } & 1.0567 & $1.0626( \pm 0.0004)$ & $1.0662( \pm 0.0001)$ & 3.3767 & $3.4081( \pm 0.0008)$ & $3.4116( \pm 0.0002)$ \\
\hline $\mathrm{M}(1)$ & & 1.0563 & $1.0623( \pm 0.0004)$ & $1.0657( \pm 0.0001)$ & 3.3765 & $3.4082( \pm 0.0008)$ & $3.4116( \pm 0.0002)$ \\
\hline $\operatorname{ICAR}(2)$ & & 1.0564 & $1.0612( \pm 0.0003)$ & $1.0652( \pm 0.0001)$ & 3.3856 & $3.4072( \pm 0.0008)$ & $3.4134( \pm 0.0003)$ \\
\hline $\mathrm{M}(2)$ & & 1.0556 & $1.0603( \pm 0.0004)$ & $\mathbf{1 . 0 6 4 4}( \pm 0.0001)$ & 3.3796 & $3.4069( \pm 0.0008)$ & $\mathbf{3 . 4 1 1 1}( \pm 0.0002)$ \\
\hline $\mathrm{A}-\mathrm{M}(2)$ & & 1.0552 & $\mathbf{1 . 0 6 0 0}( \pm 0.0004)$ & $\mathbf{1 . 0 6 4 4}( \pm 0.0001)$ & 3.3799 & $\mathbf{3 . 4 0 6 7}( \pm 0.0008)$ & $\mathbf{3 . 4 1 1 1}( \pm 0.0002)$ \\
\hline $\operatorname{ICAR}(1)$ & \multirow{5}{*}{$-\mathrm{IGN}$} & 1.9964 & $2.0022( \pm 0.0002)$ & $2.0054( \pm 0.0001)$ & 3.1773 & $3.1863( \pm 0.0002)$ & $3.1875( \pm 4 \mathrm{E}-05)$ \\
\hline $\mathrm{M}(1)$ & & 1.9964 & $2.0022( \pm 0.0002)$ & $2.0054( \pm 0.0001)$ & 3.1773 & $3.1863( \pm 0.0002)$ & $3.1875( \pm 4 E-05)$ \\
\hline $\operatorname{ICAR}(2)$ & & 1.9958 & $2.0004( \pm 0.0002)$ & $2.0043( \pm 0.0001)$ & 3.1801 & $3.1862( \pm 0.0002)$ & $3.1885( \pm 7 \mathrm{E}-05)$ \\
\hline $\mathrm{M}(2)$ & & 1.9957 & $2.0003( \pm 0.0002)$ & $\mathbf{2 . 0 0 4 2}( \pm 0.0001)$ & 3.1783 & $3.1861( \pm 0.0002)$ & $\mathbf{3 . 1 8 7 5}( \pm 4 \mathrm{E}-05)$ \\
\hline $\mathrm{A}-\mathrm{M}(2)$ & & 1.9953 & $1.9999( \pm 0.0002)$ & $\mathbf{2 . 0 0 4 2}( \pm 0.0001)$ & 3.1784 & $\mathbf{3 . 1 8 6 0}( \pm 0.0002)$ & $\mathbf{3 . 1 8 7 5}( \pm 4 \mathrm{E}-05)$ \\
\hline $\operatorname{ICAR}(1)$ & \multirow{5}{*}{ INT } & 8.7584 & $8.8049( \pm 0.0030)$ & $8.8324( \pm 0.0007)$ & 27.890 & $28.131( \pm 0.0068)$ & $28.161( \pm 0.0019)$ \\
\hline $\mathrm{M}(1)$ & & 8.7729 & $8.8193( \pm 0.0042)$ & $8.8443( \pm 0.0013)$ & 27.889 & $28.131( \pm 0.0068)$ & $28.161( \pm 0.0019)$ \\
\hline $\operatorname{ICAR}(2)$ & & 8.7651 & $8.8034( \pm 0.0029)$ & $8.8357( \pm 0.0009)$ & 27.952 & $28.121( \pm 0.0069)$ & $28.207( \pm 0.0041)$ \\
\hline $\mathrm{M}(2)$ & & 8.7536 & $\mathbf{8 . 7 8 9 7}( \pm 0.0029)$ & $\mathbf{8 . 8 2 4 3}( \pm 0.0007)$ & 27.909 & $28.120( \pm 0.0069)$ & $28.163( \pm 0.0019)$ \\
\hline $\mathrm{A}-\mathrm{M}(2)$ & & 8.7532 & $8.7914( \pm 0.0034)$ & $8.8277( \pm 0.0007)$ & 27.911 & 28.118( $( \pm 0.0069)$ & $28.163( \pm 0.0019)$ \\
\hline
\end{tabular}

\section{References}

Cryer, J. D. and Chan, K.-S. (2008). Time series analysis with applications in $R$. Springer, New York, NY, second edition. 9

Petersen, K. B. and Pedersen, M. S. (2012). The matrix cookbook. Version 20121115. 3,5 
Sidén, P., Eklund, A., Bolin, D., and Villani, M. (2017). Fast Bayesian whole-brain fMRI analysis with spatial 3D priors. NeuroImage, 146:211-225. 1, 2, 7

Simpson, D. P., Rue, H., Riebler, A., Martins, T. G., and Sørbye, S. H. (2017). Penalising model component complexity: A principled, practical approach to constructing priors. Statistical Science, 32(1):1-28. 7 